near the tips of the pyramids. They attributed the location of the nucleation sites to the unique strain gradients as a direct result of the template geometry. They said that superlattice instability and compressive strain, due to dissimilar coefficients of thermal expansion, could also favor nucleation. This feature, the researchers suggest, could be used in the fabrication of large arrays of individually addressable silicon quantum dots. The researchers were able to show that Si nanocrystals could be placed in specific locations on a Si wafer by using standard Si processing techniques.

CALIN Miclaus

\section{Theoretical Study Predicts Amide Formation is Preferred on Armchair Single-Walled Carbon Nanotubes}

Using a theoretical approach to study the reactivity of carboxylic groups toward amines in the direct amidation of singlewalled carbon nanotubes (SWNTs) and its dependence on the nanotube structure, some aspects of the chemical derivatization of these nanotubes have become further understood and their application areas expanded. Application areas of nanotubes could be significantly expanded by chemical derivatization. As reported in the November issue of Nano Letters, researchers Vladimir Basiuk, Elena Basiuk, and José-Manuel Saniger-Blesa of the National Autonomous University of Mexico obtained theoretical evidence that the direct formation of amides proceeds preferentially on armchair SWNT tips as compared with zigzag SWNT tips, according to the scheme

$$
\begin{aligned}
& \text { SWNT-COOH }+\mathrm{HNR}^{1} \mathrm{R}^{2} \rightarrow \\
& \text { SWNT-CO-NR }{ }^{1} \mathrm{R}^{2}+\mathrm{H}_{2} \mathrm{O},
\end{aligned}
$$

where $\mathrm{R}^{1}=\mathrm{H}$ or Alk, and $\mathrm{R}^{2}=\mathrm{Alk}$.

The reaction mechanisms were studied using a two-level so-called ONIOM (own n-layered integrated molecular orbital and molecular mechanics) approach, where the molecular system being studied is divided into two layers that are treated with different model chemistries. The results are then automatically combined into the final predicted results. Monocarboxylated short fragments of $(10,0)$ zigzag SWNTs and $(5,5)$ armchair SWNTs were used to interact with methylamine as model reaction systems. The transition states of the molecular systems were also treated and followed using ONIOM refinement. The researchers reported that all geometric optimizations follow the default convergence criteria set in the Gaussian 98W program.

According to the report, analysis of total optimized geometries for the reaction complexes shows that there is a weak hydro- gen bond between the amino and carboxylic groups in the armchair SWNTs, whereas the corresponding distance in the zigzag nanotubes is far too long to consider it a $\mathrm{H}$ bond. The transition states in both models do not differ significantly in geometry, but the energy of the armchair reaction complex is $10.5 \mathrm{kcal} \mathrm{mol}-1$ higher than that of its zigzag counterpart, implying, the researchers said, that there is a stronger methylamine adsorption on the zigzag SWNTs. Also, the armchair amidation products achieve a lower energy compared with the zigzag SWNT tips; therefore, the latter reaction is less energetically favorable, according to the researchers.

While the results of two-level ONIOM calculations suggest that the direct amidation of carboxylic groups on the armchair SWNT tips is energetically preferable to the zigzag nanotube tips, Basiuk said, "Further detailed studies are desirable to verify whether this can be generalized for other aliphatic amines....It is too premature to discuss practical implications of the data obtained."

KINSON C. KAM

\section{Ferroelectric Domain Inversion Demonstrated in $\mathrm{BaMgF}_{4}$}

In their study of $\mathrm{BaMgF}_{4}(\mathrm{BMF})$ as a material for ultraviolet generation, researchers from the Massachusetts Institute of Technology, AC Materials (Winter Park, Fla.), and the National Institute of Standards and Technology have demonstrated ferroelectric domain inversion. It also has transparency to $<140 \mathrm{~nm}$, and is resistant to solarization. $\mathrm{BMF}$, along with $\beta-\mathrm{BaB}_{2} \mathrm{O}_{4}, \mathrm{LiB}_{3} \mathrm{O}_{5}$, and $\mathrm{CsLiB}_{6} \mathrm{O}_{10}$, displays short-wavelength transparency and the potential for UV and vacuum-UV frequency conversion.

To achieve frequency conversion in materials, phase-matching has to be obtained first. One option researchers have is to create materials with a large birefringence, which is not applicable in the case of BMF. Another possibility is to use ferroelectric domain inversion techniques to produce a quasi-matched structure. The advantage of quasi-phase matching (QPM) is that the output wavelength is a function of the period of the structure and is independent of the material's parameters.

According to the researchers' report in the November 1 issue of Optics Letters, BMF single-domain crystals, in the shape of an elongated sphere (with the longest dimension of $3 \mathrm{~cm}$ along the growth direction and a maximum diameter of $2 \mathrm{~cm}$ ), were grown using the Czochralski technique. Based on the transmission spectra, the BMF crystals were transparent to wavelengths of $\leq 140 \mathrm{~nm}$. Refractive indices of interest were measured and correlated with the values given by the Sellmeier fit. The results were then used to calculate the grating periods for QPM interactions. Periodically poled BMF was fabricated from a 500- $\mu$-thick crystal, lithographically patterned with a $19.2-\mu \mathrm{m}$ grating. A charge of $5.4 \mu \mathrm{C}$ was delivered in 19 pulses at voltages from $16 \mathrm{kV} / \mathrm{mm}$ to $20 \mathrm{kV} / \mathrm{mm}$. The domains were imaged with an environmental scanning microscope. According to the researchers, this experiment demonstrated short-wavelength transmission, domain inversion, and solarization resistance in $\mathrm{BMF}$.

IULiA MunTELE

\section{Narrow-Band Čerenkov Radiation Generated in a Silicon Foil}

By firing 5-MeV electrons at a 10- $\mathrm{mm}$ thick silicon foil, researchers in the Netherlands have succeeded in generating narrow-band Čerenkov radiation at $99.7 \mathrm{eV}$, proving that soft $\mathrm{x}$-rays (in the range of $100 \mathrm{eV}$ to several $\mathrm{keV}$ ) can be produced using small accelerators and moderate electron energies. The emission of Čerenkov radiation occurs when the velocity of a charged particle exceeds the phase velocity of light $(c / n)$, where $c$ is the velocity of light and $n$ is the refractive index of the medium. This requires $n>1$, but at ultraviolet and shorter wavelengths, $n$ is generally less than 1 . However, there are narrow regions of resonant anomalous dispersion at atomic absorption edges for which $n>1$ at soft x-ray wavelengths. Previous demonstrations of this radiation phenomenon used electrons with energies of at least $75 \mathrm{MeV}$; this demonstration used moderate-energy electrons, as reported in the October 29 issue of Applied Physics Letters.

W. Knulst and co-workers at the Eindhoven University of Technology, and J. Verhoeven of the FOM Institute for Atomic and Molecular Physics in Amsterdam, used a multilayered mirror consisting of 101 alternating layers of Mo and Si coupled with a photodiode to detect the Čerenkov radiation. Electrons accelerated to $5 \mathrm{MeV}$ passed through the $10-\mu \mathrm{m}$ foil of undoped single-crystal silicon and were bent by a $90^{\circ}$ dipole magnet into an electron dump. The Čerenkov radiation from the silicon $L$ edge was emitted in a cylindrical cone around the original electron trajectory in the forward direction. For a silicon foil and 5-MeV electrons, the angle of maximum emission of radiation was calculated to be $10.6^{\circ}$, so the detector geometry was arranged to measure the yield at emission angles of $5^{\circ}$ and $10^{\circ}$ $\left( \pm 0.5^{\circ}\right)$. A yield of $1 \times 10^{-3}$ photon/electron was obtained, which is in reasonable 